\title{
Validation List no. 93 Valid publication of new names and new
combinations previously effectively published
outside the IJSEM
}

Correspondence Jean Euzéby j.euzeby@envt.fr
The purpose of this announcement is to effect the valid publication of the following new names and new combinations under the procedure described in the Bacteriological Code (1990 Revision). Authors and other individuals wishing to have new names and/or combinations included in future lists should send three copies of the pertinent reprint or photocopies thereof to the IJSEM Editorial Office for confirmation that all of the other requirements for valid publication have been met. It is also a requirement of IJSEM and the ICSP that authors of new species, new subspecies and new combinations provide evidence that types are deposited in two recognized culture collections in two different countries (i.e. documents certifying deposition and availability of type strains). It should be noted that the date of valid publication of these new names and combinations is the date of publication of this list, not the date of the original publication of the names and combinations. The authors of the new names and combinations are as given below, and these authors' names will be included in the author index of the present issue and in the volume author index. Inclusion of a name on these lists validates the name and thereby makes it available in bacteriological nomenclature. The inclusion of a name on this list is not to be construed as taxonomic acceptance of the taxon to which the name is applied. Indeed, some of these names may, in time, be shown to be synonyms, or the organisms may be transferred to another genus, thus necessitating the creation of a new combination.

\begin{tabular}{|c|c|c|c|c|}
\hline Name/author(s) & Proposed as: & Nomenclatural type ${ }^{\star}$ & Priority $\dagger$ & Reference \\
\hline Anoxynatronum sibiricum Garnova et al. 2003 & sp. nov. & Strain Z-7981 (=DSM $15060=$ Uniqem U218 $)$ & 6 & 3 \\
\hline Enhygromyxa Iizuka et al. 2003 & gen. nov. & Enhygromyxa salina Iizuka et al. 2003 & 7 & 4 \\
\hline Enhygromyxa salina Iizuka et al. 2003 & sp. nov. & Strain SHK-1 (=JCM $11769=$ DSM 15217) & 7 & 4 \\
\hline Gordonia sinesedis Maldonado et al. 2003 & sp. nov. & Strain J72 (=DSM $44455=$ NCIMB 13802) & 4 & 6 \\
\hline Salinisphaera shabanensis Antunes et al. 2003 & sp. nov. & Strain E1L3A (=DSM $14853=\mathrm{JCM} 11575)$ & 9 & 1 \\
\hline $\begin{array}{l}\text { Staphylococcus equorum subsp. linens Place } \\
\text { et al. 2003॥ }\end{array}$ & subsp. nov. & Strain RP29 (=DSM 15097=CIP 107656) & 2 & 8 \\
\hline Streptacidiphilus Kim et al. 2003 & gen. nov. & Streptacidiphilus albus Kim et al. 2003 & 1 & 5 \\
\hline Streptacidiphilus albus Kim et al. 2003 & sp. nov. & Strain JL83 (=DSM 41753= KCTC 9910) & 1 & 5 \\
\hline Streptomyces sanglieri Manfio et al. 2003 & sp. nov. & Strain A46R51 ( = DSM 41791= NCIMB 13929) & 5 & 7 \\
\hline Streptomyces yatensis Saintpierre et al. 2003 & sp. nov. & Strain SFOCin $76(=$ DSM $41771=$ NRRL B-24116) & 3 & 9 \\
\hline
\end{tabular}

For references to Validation Lists 1-71, see Int J Syst Bacteriol 49 (1999) 1325. Lists 72-92 were published in Int J Syst Evol Microbiol 50 (2000) 3, 423, 949, 1415, 1699, 1953 and 51 (2001) 1, 263, 793, 1229, 1619, 1945 and $\mathbf{5 2}$ (2002) 3, 685, 1075, 1437, 1915 and 53 (2003) 1, $373,627,935$. 
*Abbreviations: ATCC, American Type Culture Collection, Manassas, VA, USA; CCUG, Culture Collection, University of Göteborg, Göteborg, Sweden; CIP, Collection of the Institute Pasteur, Paris, France; DSM, DSMZ-Deutsche Sammlung von Mikroorganismen und Zellkulturen, Braunschweig, Germany; JCM, Japan Collection of Microorganisms, RIKEN, Saitama, Japan; KCTC, Korean Collection for Type Cultures, Korea Research Institute of Bioscience \& Biotechnology, Yusong, Taejon, Republic of Korea; NCIMB, National Collection of Industrial and Marine Bacteria, Aberdeen, UK; NRRL, Agricultural Research Service Culture Collection, National Center for Agricultural Utilization Research, US Department of Agriculture, Peoria, IL, USA; Uniqem, Collection of Unique Cultures, Russian Academy of Sciences, Moscow, Russia; WAL, Wadsworth Anaerobe Laboratory, Wadsworth Hospital Center, Los Angeles, CA, USA.

$\dagger$ Priority number assigned according to the date the documentation and request for validation are received.

$\ddagger$ Culture collection accession number provided on request for validation.

\$An emended description of the genus Cetobacterium is also provided. (i.e. Cetobacterium Foster et al. 1996 emend. Finegold et al. 2003).

IIAccording to Rule 40d (formerly Rule 46) of the Bacteriological Code (1990 Revision), the valid publication of Staphylococcus equorum subsp. linens Place et al. 2003 automatically creates another subspecies, Staphylococcus equorum subsp. equorum Schleifer et al. 1985.

$\uparrow$ An emended description of the family Streptomycetaceae is also provided [i.e. Streptomycetaceae Waksman and Henrici 1943 (Approved Lists 1980) emend. Kim et al. 2003].

\section{References}

1. Antunes, A., Eder, W., Fareleira, P., Santos, H. \& Huber, R. (2003). Salinisphaera shabanensis gen. nov., sp. nov., a novel, moderately halophilic bacterium from the brine-seawater interface of the Shaban Deep, Red Sea. Extremophiles 7, 29-34.

2. Finegold, S. M., Vaisanen, M. L., Molitoris, D. R., Tomzynski, T. J., Song, Y., Liu, C., Collins, M. D. \& Lawson, P. A. (2003). Cetobacterium somerae sp. nov. from human feces and emended description of the genus Cetobacterium. Syst Appl Microbiol 26, 177-181.

3. Garnova, E. S., Zhilina, T. N., Tourova, T. P. \& Lysenko, A. M. (2003). Anoxynatronum sibiricum gen. nov., sp. nov. alkaliphilic saccharolytic anaerobe from cellulolytic community of Nizhnee Beloe (Transbaikal region). Extremophiles 7, 213-220.

4. lizuka, T., Jojima, Y., Fudou, R., Tokura, M., Hiraishi, A. \& Yamanaka, S. (2003). Enhygromyxa salina gen. nov., sp. nov., a slightly halophilic myxobacterium isolated from the coastal areas of Japan. Syst Appl Microbiol 26, 189-196.

5. Kim, S. B., Lonsdale, J., Seong, C. N. \& Goodfellow, M. (2003). Streptacidiphilus gen. nov., acidophilic actinomycetes with wall chemotype I and emendation of the family Streptomycetaceae (Waksman and Henrici (1943) ${ }^{\mathrm{AL}}$ ) emend. Rainey et al. 1997. Antonie van Leeuwenhoek 83, 107-116.

6. Maldonado, L. A., Stainsby, F. M., Ward, A. C. \& Goodfellow, M. (2003). Gordonia sinesedis sp. nov., a novel soil isolate. Antonie van Leeuwenhoek 83, 75-80.

7. Manfio, G. P., Atalan, E., Zakrzewska-Czerwinska, J., Mordarski, M., Rodríguez, C., Collins, M. D. \& Goodfellow, M. (2003). Classification of novel soil streptomycetes as Streptomyces aureus sp. nov., Streptomyces laceyi sp. nov. and Streptomyces sanglieri sp. nov. Antonie van Leeuwenhoek 83, 245-255.

8. Place, R. B., Hiestand, D., Gallmann, H. R. \& Teuber, M. (2003). Staphylococcus equorum subsp. linens, subsp. nov., a starter culture component for surface ripened semi-hard cheeses. Syst Appl Microbiol 26, 30-37.

9. Saintpierre, D., Amir, H., Pineau, R., Sembiring, L. \& Goodfellow, M. (2003). Streptomyces yatensis sp. nov., a novel bioactive streptomycete isolated from a New-Caledonian ultramafic soil. Antonie van Leeuwenhoek 83, 21-26. 